\title{
Retention of phonemic and semantic features of words
}

\author{
LAIRD S. CERMAK and CAROLYN P. YOUTZ \\ Psychology Service, Boston Veterans Administration Hospital, Boston, Massachusetts 02130 \\ and \\ Aphasia Research Unit, Neurology Department, Boston University School of Medicine \\ Boston, Massachusetts 02215
}

\begin{abstract}
Subjects were asked to detect either repetitions, rhymes, or words from the same category during an auditory or visual list presentation. It was discovered in both cases that the number of intervening words had a differential effect on probability of detection, with phonemic feature detection deteriorating more rapidly than semantic. However, "rate" of presentation did not have a differential effect on the probability of feature detection, since phonemic and semantic feature detection improved equally with increased interword interval time. It was suggested that phonemic feature retention might be more vulnerable than semantic feature retention to interference.
\end{abstract}

There is now a good deal of evidence to support the theory that a more permanent memory trace is established when a subject concentrates on the meaning of a word than when he concentrates on the sound of a word (Cermak, Schnorr, Buschke, \& Atkinson, 1970; Jacoby \& Goolkasian, 1973). Several theorists have tried to explain this phenomenon by hypothesizing, first, that phonemic analyses result in memory traces consisting mainly of phonemic features and semantic analyses result in memory traces consisting mainly of semantic features, and, second, that the representation of phonemic features in memory deteriorates faster than the representation of semantic features (Craik \& Lockhart, 1972; Kintsch \& Buschke, 1969). Unfortunately, two separate attempts to distinguish differential rates of decay for phonemic and semantic features have not been successful (Bregman, 1968; Shulman, 1970). However, in both these experiments the subject was encouraged to encode each word as fully as possible either because he did not know which feature he would be asked to recognize (Shulman) or because he knew tinat recall of the word would be required and not just one feature of the word (Bregman).

In order to encourage subjects to analyze specific features of a word, and to rely upon this analysis, Jacoby (1974) developed a procedure he called the "looking-back" technique. In this procedure, the subject is asked to look at each word in a list and to indicate whether or not a previously presented word shares a particular predetermined feature with that word. Decay of feature representation can then be determined by the change in probability of detecting a feature match when the number of intervening words

This research was supported in part by NIH Grant AA-00187 to Boston University School of Medicine. Reprint requests should be sent to Laird S. Cermak, Psychology Service, Boston Veterans Administration Hospital, 150 South Huntington Avenue, Boston, Massachusetts 02130 . is increased. Nevertheless, despite these improvements in procedure. Jacoby still found equal rates of decay for phonemic and semantic feature representation. However, there was one potentially contaminating feature of this procedure in that the rhyming words not only sounded alike but looked alike as well. In fact, only the initial letters were changed ("rock" to "dock"), and consequently the subject may have been recognizing repeated visual displays rather than repeated "sounds." In order to prevent this possibility in the present investigation, the "looking-back" technique was employed, first with an auditory presentation (Experiment I) and second with a visual presentation in which the rhyming words did not also look alike (Experiment II). In addition, two rates of presentation were used in Experiment $I$ in order to further assess the possibility that phonemic features might decay over time faster than semantic features. Experiments I and II also included a repetition detection task in order to determine the maximum performance level for the "looking-back" task when subjects are not restricted to analysis on the basis of just one feature of the information.

\section{EXPERIMENT I}

\section{Method}

Subjects. The subjects were 120 in troductory psychology students at Tutts University who chose to participate in the experiment for course credit. The subjects were assigned in the order of their appearance to one of two groups. One group $(n=60)$ heard words read at the rate of 1 word/sec and the other group ( $n=60$ ) heard words read at the rate of 1 word $/ 3 \mathrm{sec}$. Otherwise, the treatment of the two groups was the same.

Task. The subject was required to listen to a recording of 45 lists of words varying in length from 6 to 18 words per list. For 15 of these lists, the subject was instructed to say "now" when he heard a word that was a repetition of a previous word in the list (repetition task). For another 15 of these lists, the subject was instructed to say "now" when he heard a word that rhymed with a previous word in the list (phonemic task). For the third block of 15 lists, the subject 
was instructed to say "now" when he heard a word that was a member of the same semantic category (example: "west." "south") as a previous word in the list (semantic task). The semantic task of detecting words from the same category was chosen rather than detecting synonyms because many words have no synonym but fall rather easily within the confines of a general category containing other words of equal frequency. The order of presentation of the three tasks was counterbalanced across subjects.

Materials. Each of the $\mathbf{4 5}$ lists presented to the subject contained two target words embedded among 4 to 16 filler words. The filler words and the repetition and phonemic target words were taken from the A or AA words in the Thorndike-Lorge word count. The semantic target words came from the norms prepared by Batrig and Montague (1969). Care was taken that the target words were not any longer. shorter, or less frequently used than the filler words.

The first target word appeared in one of three possible positions-the third, the fitth. or the seventh position in the list. The second target word then occurred in one of five positions-the first. the third, the fifth, the seventh, or the ninth position after the tirst target word. This made a total of 15 different combinations of first and second target-word positions. The second target word was always followed by two more filler words so that the subject would not simply respond to the end of the list. No subject heard the same nord twice unless it was a repetition target word.

There were six possible orders in which the three tasks could be given to the subject: (1) repetition, phonemic, semantic; (2) repetition. semantic, phonemic; (3) phonemic, repetition. semantic; (4) .... etc. All six orders were used, and each order was given to 10 subjects in each group.

For each task, the subject heard a block of 15 lists of words. Each block of 15 lists contained all 15 combinations of first and second target-word positions. There were 15 possible orders in which these 15 combinations could be presented to the subject. From all possible orders. a set of 15 was chosen such that each combination could be placed in a different position across sets. Then the 15 orders were each used for eight subjects (four subjects in each group) for each of the tasks. This procedure thus counterbalanced the effects of order of presentation of the combination across subjects. The same procedure was also followed to assign the actual target word pairs to the combinations counterbalancing the possible effects of variable difficulty of target-word pairs within tasks.

Apparatus and Procedure. The subject was seated in a small testing room across a table from the experimenter. The subject was informed that he would be hearing prerecorded word lists presented on a Sony cassette tape recorder and that his task would be to detect a repetition (or rhyme or word from the same semantic category). He was told that when he heard this particular item he was to respond with the word "now," indicating that he had detected the target word. Half the subjects $(60)$ heard the words read at a 1-word/sec rate, while the other half heard the same words read at a 1-word 3 -sec rate. After the instructions were given, the subject heard all 15 lists of his first task. Each list was separated by $5 \mathrm{sec}$ and the announcement of the number of the next list. Tasks were separated by a break of $5 \mathrm{~min}$. and during the break the subject was given instructions for his next block of 15 lists.

Data analysis. In order to perform an analysis of variance on the binary data of this experiment, several subjects had to be combined. Then the data for each of these "supersubjects" (six subjects each) could be analyzed. The number six was chosen so that each "supersubject" would include all six orders of presentation of the three tasks.

\section{Results}

Since the trend in results for all three initial target-word positions was very nearly identical, these data were combined for all analyses. It must be noted, however, that performance overall was the best when the initial target word appeared early in the list and that it fell somewhat when this word was delayed.

As can be seen in Figure 1, performance on all three tasks decreased as the number of intervening words between target items increased, $F(4,72)=$ 183.48, $\mathrm{p}<.001$. MSE $=1.13$. This decrease was most notable for the detection of rhymes, since performance on this task was as good as detection of repetitions when no items intervened between target items, but fell rapidly as soon as two intervening items appeared and became progressively worse as more items were added. On the other hand, detection of words from the same semantic category was worse than both repetition and rhyme detection when the target items were adjacent (indicating perhaps that the task was more difficult than the other two tasks), but deteriorated to a far less extent than rhyme detection. In fact, as soon as two items intervened between the target words. category detection became superior to. and remained above, rhyme detection. Statistically. these trends are represented by an effect of tasks. $F(2.36)=273.92, p<.001, \mathrm{MSE}=1.59$, and a Task by Number of Intervening Words interaction, $\mathrm{F}(8.144)=27.56, \mathrm{p}<.001, \mathrm{MSE}=.91$.

Performance on all three tasks also improved when the rate of presentation was slowed. as can be seen by
A

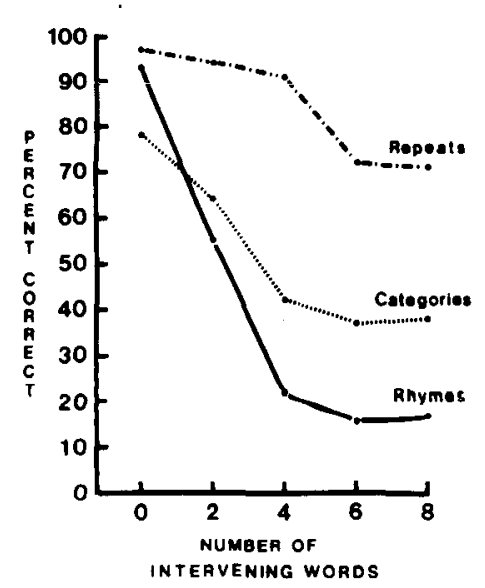

B

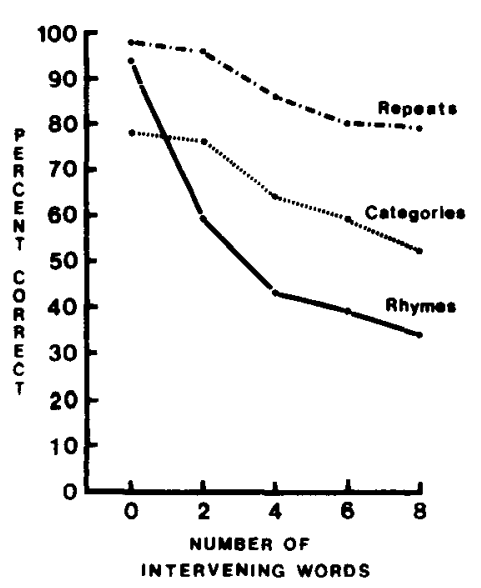

Figure 1. Percentage of target worda correctly identified for each of the three tasks (repetitions, phonemic-rhymes, and semanticcategories) as a function of the number of intervening words when the words were presented auditorially (Experiment D. Part A presents the results when the material was presented at a 1-word/sec rate and Part $B$ at a 1-word/3-sec rate. 
contrasting Part A (1 word/sec) with Part B (1 word $3 \mathrm{sec})$ of Figure 1, $F(1,18)=88.81$. $\mathrm{p}<.001, \mathrm{MSE}=.99$. Rate also interacted with task. $F(2,36)=7.77, p<.001, \mathrm{MSE}=1.59$, such that the difference between the repeat condition and the other two conditions was larger at the fast than at the slow rate (see Figure 1). However, this interaction was not significant when only the rhyme and category detection tasks were considered. In fact, when analysis was restricted to performance on these two tasks alone, the only significant interaction was Task by Number of Intervening Words, $F(4,72)=21.51$, $\mathrm{p}<.001, \mathrm{MSE}=1.02$. Thus, the detection of rhymes was more affected by the number of intervening words than was category detection (see Figure 1).

\section{EXPERIMENT II}

Experiment I demonstrated subjects' differential ability to detect phonemic and semantic feature similarity following two or more intervening words. However, since this experiment presented the material auditorially, rather than visually as in the Jacoby (1974) experiment, the possibility existed that mode of presentation might somehow have produced this outcome. Consequently, it was necessary to replicate Experiment $I$ in the visual modality, taking measures to assure that rhyming words were dissimilar visually (e.g., news, lose). This would prevent the possibility that phonemic similarities could be detected simply on the basis of shared physical features (e.g., sight. might).

\section{Method}

Subjects. Sixty volunteer subjects from the same population as that represented in Experiment I participated in this experiment. None of these subjects had taken part in Experiment I.

Task. The task was as it had been in Experiment $I$ except that the subject was now asked to look at 45 lists of words (6-18 words per list l presented one word at a time on a memory drum. For 15 of these lists. the subject was asked to say "now" when he saw a word repeated (repetition task), for another 15 lists he was asked to detect rhymes (phonemic task). and for still another 15 lists he was asked to detect words from the same category (semantic task). The order of presentation of the three tasks was again counterbalanced.

Materials. The same lists used in Experiment $I$ were used in Experiment II, except that 10 of the 15 rhyming pairs were discarded and replaced with pairs that were visually dissimilar. In all other respects, construction of the lists was precisely as it had been in Experiment I.

Apparatus and Procedure. The subject was seated in a small testing room in front of a table containing a Lafayette automatic memory drum. He was told that he would be seeing lists of words and that his task would be to detect a repetition (or rhyme or word from the same semantic category). All the subjects saw the words presented at a 1-word/sec rate, with each list separated by 5 sec. Tasks were separated by 5 min. during which the subject was given instructions for the next task. Analysis of the data was again based upon the "supersubjects." with six subjects per unit of analysis.

\section{Results}

The results of Experiment II (shown in Figure 2) were very nearly identical to those of Experiment $I$.

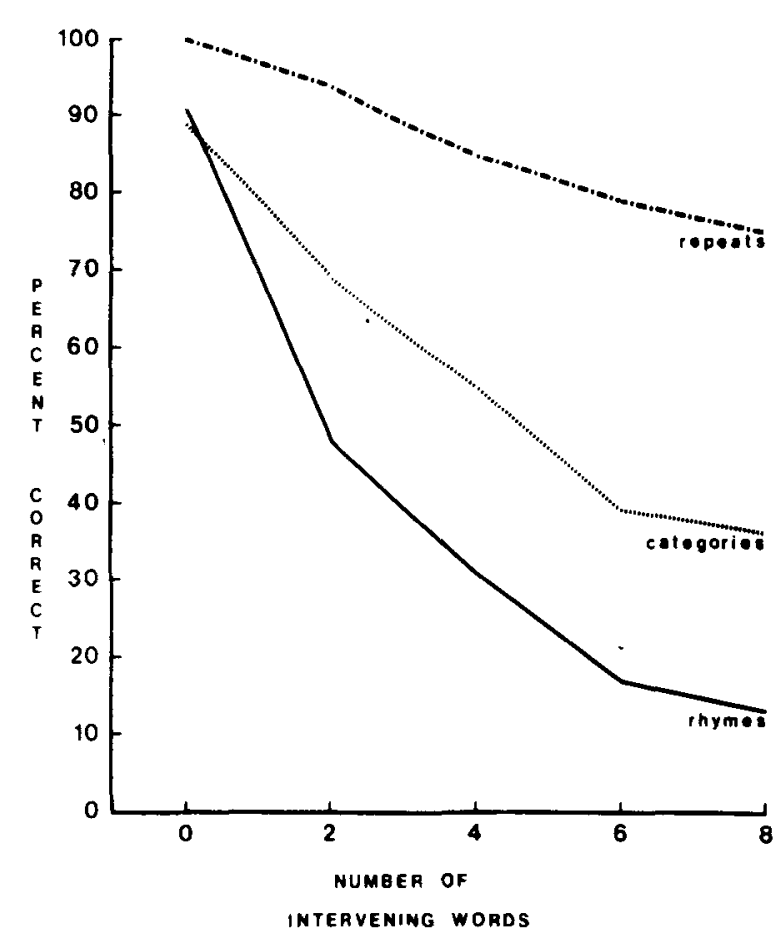

Figure 2. Percentage of target words correctly identified for each of the three tasks (repetitions, phonemic-rhymes, and semanticcategories) as a function of the number of intervening words when the words were presented visually (Experiment II).

Performance on all three tasks deteriorated as the number of intervening items increased. $F(4.36)=$ 153.95. $\mathrm{p}<.001$. MSE $=1.09$. However. performance on the repetition task was superior to that of category detection which, in turn, exceeded that of the rhyme detection as soon as two or morc items $i_{1}$, tervened between target words. $F(2,18)=$ 208.44. $\mathrm{p}<.001 . \mathrm{MSE}=1.60$. The Task by Number of Intervening Words interaction was again significant. $F(8,72)=16.05, p<.001$, MSE $=.78$. indicating (as can be seen in Figure 2) that the repetition task was least affected by the number of intervening words, while the phonemic task was most affected. When the repetition task was excluded from the analysis. precisely the same results were obtained.

\section{DISCUSSION}

The hypothesis that reliance upon phonemic analysis would result in a more rapid loss of feature representation than would be the case for semantic analyses was contirmed by the results of this study. Detection on the basis of both these dimensions progressively deteriorated as the number of items intervening between target words increased. However. this deterioration was most dramatic for the detection of rhymes whether the material was presented auditoriaily (Figure 1) or visually (Figure 2). Apparently, the moditications made to Jacoby's "looking-back" technique, namely either presenting the material auditorially or insuring that sound-alike 
words do not also look alike when presented visually. were successful in persuading the subject to rely upon his analysis of specific features of words.

The results of this investigation support the notion that phonemic feature representations dissipate from memory faster than semantic feature representations (Craik \& Lockhart, 1972; Kintsch \& Buschke, 1969). Thus, the observation that more permanent memory traces are formed when a subject concentrates on the meaning. rather than the sound. of a word can be explained in terms of differential rates of feature loss. In order to assess whether or not this differential feature loss was due simply to different rates of feature "decay" over time, Experiment 1 included two rates of presentation of the material. It was hypothesized that if phonemic features decay faster than semantic features. then the difference in the probability of detecting semantic and phonemic feature similarities would be magnified as the rate of presentation was slowed. However, it turned out that neither feature detection task suffered from this decrease in presentation rate; rather, each protited. and to essentially the same degree. This means that the differential loss of phonemic and semantic feature representation, apparent throughout this investigation. wás probably not due to different decay rates. Instead, a plausible alternative explanation for the finding might be that phonemic and semantic features are differentially vulnerable to interference. This could mean that uninterrupted phonemic traces would remain as stable as uninterrupted semantic traces, but further investigations will be needed to substantiate this hypothesis.

\section{REFERENCES}

Battic. W. F. \& Montague. W. E. Category norms for serbal items in 56 categories: $A$ replication and extension of the Connecticut category norms. Journal of Expenmental Psichology. 1969. 8013. Pt. 2).

Bregman. A. S. Forgetting curves with semantic. phonetic. graphic and contiguity cues. Journal of Experimentul Psichology, 1968, 78, 539-546.

Cermak. G., Schnorr. J.. Buschke, H., \& Atkinson. R. C Recognition memory as influenced by differential attention to semantic and acoustic properties of words. Psychonomic Science, 1970, 19, 79-81.

Craik, F. I. M., \& Lockhart, R. S. Leveis of processing: A framework for memory research. Journal of Verbal Learning and Verbal Behavior, 1972, 11. 671-684.

$J_{A C O B Y}$ L. L. The role of mental contiguity in memory: Registration and retrieval eifects. Journal of Verbal Learning and Verbal Behavior, 1974, 13, 483-496.

JaC OBY, L. L., \& Goolkasian. P. Semantic versus acoustic coding: Retention and conditions of organization. Journal of Verbal Learning and Verbal Behavior, 1973, 12, 324-333.

Johnston, C. D.. \& Jenkins, J. J. Two more incidental tasks that differentially effect associate clustering in recall. Journal of Experimental Psychology, 1971, 89, 92-95.

Kintsch, W.. \& Buschke. H. Homophones and synonyms in short-term memory. Journal of Experimental Psychology. 1969, 80, 403-407.

Shulman. H. G. Encoding and retention of semartic and phonemic information in short-term memory. Journal of Verbal Le'aming and Verbal Behavior, 1970, 9. 499-508.

(Received for publication February 12, 1975; revision received June 18.1975 .) 